\section{Menor Incidência de Hipoglicemia Noturna com o Uso de Insulina Lispro Comparada à Insulina Humana Regular no Tratamento de Pacientes com Diabetes do Tipo 1}

artigo original

Bernardo L. Wajchenberg Antônio R. Chacra Adriana C. Forti* Sandra R.G. Ferreira Odette de Oliveira Carla F. Lopes Antonio C. Lerário Rita de Cássia Sena Marcia J. Kayath

* pelo Grupo do Centro Integrado de Diabetes e Hipertensão, Universidade Federal do Ceará (Socorro S. Gomes, Margarida M. Oliveira, Adélia M.A. Bandeira, Marta T. Moreira, Vanesca F. Ribeiro)

Unitermos: Diabetes tipo 1; Insulina lispro; Hipoglicemia; Tratamento diabetes

\section{ABSTRACT}

Insulin lispro is a human insulin analog of rapid onset of action and duration, which mimics the physiological insulin profile after a meal. We have evaluated the safety and efficacy of lispro insulin in comparison with human regular insulin in a crossover, multicenter, randomized trial in 25 type 1 diabetic patients in use of human NPH and regular insulin (median age $=16$ years). After administration of lispro or regular insulin for 2 months, the patients were transferred for the other insulin for two more months, maintaining the basal NPH insulin regimen. There was no difference in the postprandial glucose excursion and glycated hemoglobin Alc comparing the 2 groups (lispro and regular). The relative percentage decrease in glycemia was significantly greater with lispro insulin after lunch, in the first phase of the study $(p<0.02)$. The total number of hypoglycemic episodes was not different comparing both groups. However, there was a significant difference in the nocturnal hypoglycemia incidence with initial administration of lispro $(p<0.05)$. With initial administration of regular insulin, there was an increase in the incidence of nocturnal hypoglycemia $(p=0.038)$, with a subsequent reduction of hypoglycemia with insulin lispro $(p=0.04)$. In the end of the study, $68 \%$ of the patients referred preference and better feeling with lispro, compared to regular insulin. Insulin lispro was a safe and efficacious option, with lower
Disciplina de Endocrinologia,

Faculdade de Medicina da

Universidade de São Paulo (BLW, CFL, ACL); Disciplina de

Endocrinologia e Metabologia,

Universidade Federal de São Paulo (ARC, SRGF, OO); Centro Integrado de Diabetes e Hipertensão,

Universidade Federal do Ceará ( $A C F, R C S)$; Laboratório Eli Lilly do Brasil (MJK)

Recebido em 01/06/99 Aceito em 20/12/99 Revisado em 26/08/99 
incidence of nocturnal hypoglycemia in type 1 diabetics. An optimization of the basal insulin regimen is necessary to improve glucose control with the use of rapid-action insulin. (Arq Bras Endocrinol Metab 2000;44/2: 133-8)

Keywords: Type 1 diabetes; Insulin lispro: Hypoglycemia; Diabetes treatment

$\mathrm{U}$ M MELHOR CONTROLE METABÓLICO em diabéticos tipo I tem sido associado a uma menor incidência de complicações crônicas (1). Este objetivo é geralmente atingido com esquema de tratamento intensivo com insulina de longa duração, associada a insulinas de curta duração (1-4). Porém, o Estudo do Controle de Diabetes e suas Complicações (DCCT) daramente demonstra que à medida que o controle da glicemia melhora, o risco rclativo de hipoglicemia aumenta (1).

Em geral a insulina humana regular é administrada 30 minutos antes das refeições, já que esta insulina tem início de ação lento (em 30 a 60 minutos) após uma injeção subcutânea. Este fato, aliado a um tempo de ação mais prolongado, pode resultar em hiperinsulinemia e hipoglicemia tardias (5). Para muitos pacientes este esquema não é conveniente e a insulina é administrada no momento da refeição, com prejuízo em sua ação e com risco de hipoglicemia tardia.

Insulina lispro é um análogo da insulina humana desenvolvida por engenharia genética pela inversão dos aminoácidos prolina e lisina nas posições 28 e 29 da cadeia beta, resultando em uma insulina com seqüência Lis(B28)Pro(B29) (6). Esta insulina tem menor tendência para auto-agregação no local de aplicação subcutânea, é absorvida mais rapidamente que a insulina humana regular, e mimetiza o perfil fisiológico da insulina em resposta a uma refeição (6). Como resultado, ela tem um início de ação rápido ( 15 minutos), atinge um pico de ação mais precocemente (I hora) e possui uma duração de ação mais curta (4 horas) (6-12). Estudos clínicos demonstraram que esta insulina monomérica tem efeitos benéficos, como a redução da incidência de episódios hipoglicêmicos (12-16) e um melhor controle glicêmico pós-prandial (17-25). Além disso, a insulina lispro deve ser administrada imediatamente ou, se necessário, após a refeição, mantendo ação eficaz (26-29).

O objetivo primário deste estudo foi comparar o efeito da insulina lispro e insulina humana regular no controle glicêmico, particularmente pós-prandial. Outros parâmetros avaliados foram: dados de segurança da insulina lispro e da insulina regular; preferência dos pacientes por insulina lispro ou insulina humana regular; controle glicêmico através da hemoglobina glicosilada $\mathrm{A}_{\mathrm{IC}}\left(\mathrm{HbA}_{\mathrm{IC}}\right) \mathrm{e}$ a incidência de episódios hipoglicêmicos nos dois esquemas terapćuticos.

\section{PACIENTES E MÉTODOS}

Este é um cstudo multicêntrico, randomizado, cruzado, aberto, comparativo, envolvendo 27 pacientes ( 19 femininos, 8 masculinos) com diabetes tipo 1 , com idade variando de 11 a 25 anos (mediana 16 anos). As instituiçǒes onde o estudo se realizou foram: Hospital das Clínicas da Faculdade de Medicina da Universidade de São Paulo/USP ( 11 pacientes); Universidade Federal de São Paulo/UNIFESP ( 8 pacientes); e Centro Integrado de Diabetes c Hipertensão/CIDH $(8$ pacientes). Todos os pacientes tinham diabetes tipo 1 (duração média 9,0 $0 \pm 5,4$ anos; variação 1,5 a 22,5 anos) e utilizavam insulina por pelo menos 12 meses. Os pacientes não tinham complicações crônicas do diabetes, exceto por I paciente que apresentava microalbuminúria. Pacientes com creatinina sérica maior que $3 \mathrm{mg} / \mathrm{dl}$ ou evidências de retinopatia rapidamente progressiva foram excluídos.

Os pacientes receberam insulina humana regular (Humulin R, Laboratório Lilly, IN, USA) c insulina humana NPH (Humulin N, Laboratório Lilly, IN, USA) por um período introdutório de 15 dias a 2 meses. Durante este periodo, insulina humana regular foi administrada antes das refeições e insulina humana NPH 1 a 2 vezes por dia, com o objetivo de otimizar o controle glicêmico do paciente. Os pacientes foram então randomizados para uso de insulina humana regular ou insulina lispro (Humalog, Laboratório Lilly, IN, USA) por 2 meses e depois transferidos para a outra insulina por mais 2 meses. As insulinas regular ou lispro foram administradas antes das refeições (insulina humana regular, 30 minutos antes e insulina lispro imediatamente antes). A insulina humana NPH foi mantida com a mesma frequiência e horário do período introdutório. O esquema do estudo está representado na figura 1 . O estudo foi aberto porque insulina regular é geralmente recomendada 30 minutos antes das refeições, enquanto insulina lispro é melhor administrada imediatamente antes das refeições, devido a seu início rápido de ação. Hipoglicemia foi definida como uma glicemia abaixo de $54 \mathrm{mg} / \mathrm{dl}$. A excursão de glicemia foi calculada subtraindo os valores de glicemias pós-prandiais dos valores pré-prandiais das refeiçôes correspondentes. O decréscimo percentual relativo da glicemia foi calculado pela seguinte fórmula: decréscimo percentual = glicemia pós-prandial - glicemia pré-prandial/glicemia pré-prandial x 100 . 


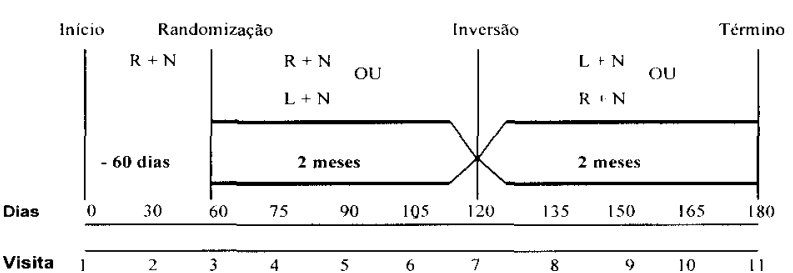

Figura 1 - Representação esquemática do estudo clínico Após um período de aproximadamente 2 meses, os pacientes eram transferidos para o outro tratamento.

$\mathrm{R}+\mathrm{N}=$ insulina regular + insulina $\mathrm{NPH}$

$L+N=$ insulina lispro + insulina $N P H$

O objetivo do estudo era atingir glicemias préprandiais entre $70-140 \mathrm{mg} / \mathrm{dl}$, glicemias pós-prandiais de 2 horas menores que $180 \mathrm{mg} / \mathrm{dl}$ c glicemias das 3 horas da madrugada maiores que $65 \mathrm{mg} / \mathrm{dl}$. Os pacientes realizaram um perfil da medida de glicemia capilar de 8 pontos, usando um glicosímetro por 2 dias não consecutivos, na semana anterior às visitas 3 , 7 e 11 . Durante estes 2 dias, em que um deveria ser de fim-de-semana, os valores de glicemia cram obtidos às 3 da madrugada, imediatamente antes e 2 horas depois das refeições da manhã, almoço, jantar e ao deitar. Hemoglobina glicosilada $\mathrm{A}_{\mathrm{IC}}\left(\mathrm{HbA}_{\mathrm{lC}}\right)$ (UNIFESP) ou hemoglobina glicada total ( $\mathrm{HbG}$ total) (USP e CIDH) foram dosadas pelo método de cromatografia por troca iônica normatizada para fração $C$, conforme a fórmula desenvolvida por um dos autores (BLW, dados não publicados): $\mathrm{HbA}_{1 \mathrm{C}}=$ $1,66+0,59 \mathrm{HbG}$ total (curva de regressão com $\mathrm{r}=$ $0,9)$. Apenas pacientes com $\mathrm{HbA}_{\mathrm{IC}}$ até 2 vezes o valor superior da normalidade foram selecionados para $o$ estudo. $\mathrm{A} \mathrm{HbA}_{\mathrm{IC}}$ foi dosada em 4 ocasiões durante o estudo (visitas 1, 3, 7 e 11 ). Um questionário ao final do estudo foi administrado pelo investigador para avaliar a preferência dos pacientes pela insulina regular ou insulina lispro. Todos os pacientes assinaram consentimento pós-informado e o estudo foi aprovado pelos comitês de ética das instituições.

\section{ANÁLISE ESTATÍSTICA}

A comparação entre variáveis quantitativas foi realizada pelos testes não paramétricos como descritos a seguir: teste de Wilcoxon entre duas visitas; teste de Friedman para mais de duas visitas e Mann-Whitney para comparação de grupos. Comparação entre as porcentagens foi realizada através do teste do chi-quadrado. Todos os valores estão expressos como média \pm desvios-padrões a não ser que indicado de outra forma. O nível de significância adotado foi um $\mathrm{p}<0,05$.

\section{RESULTADOS}

Quinze pacientes foram randomizados para uso inicial de insulina regular e 12 pacientes iniciaram o estudo com insulina lispro. As características dos 2 grupos de pacientes no início do estudo não foram significantemente diferentes e estão representadas na tabela $l$.

A dose de insulina NPH foi progressivamente aumentada no período pré-randomização do estudo $(46,7 \pm 14 \mathrm{U} /$ dia na visita $\mathrm{l}$ vs. $50 \pm 15 \mathrm{U} /$ dia na visita 3 ; $\mathrm{p}=0,02$ ). Durante todo o estudo houve uma tendência de aumento da dose da insulina NPH em ambos os grupos, sendo significante durante o período inicial do estudo com o grupo em uso inicial de lispro $(\mathrm{p}=0,03)$. A dose de insulina lispro ou regular não variou no grupo que iniciou o estudo com insulina regular, enquanto a dose de insulina lispro foi progressivamente aumentada no grupo que iniciou o estudo com insulina lispro (tabela 2). A dose de insulina regular se manteve estável

Tabela 1 - Características dos 2 grupos de pacientes na randomização do estudo".

\begin{tabular}{lcc}
\hline & Grupo 1 & Grupo 2 \\
\hline Idade (anos) & 16,8 & 15 \\
IMC (kg/m²) & $22 \pm 2,5$ & $22 \pm 3,0$ \\
Tempo de diabetes (anos) & $10,5 \pm 6,6$ & $7,6 \pm 3,6$ \\
No de episódios hipoglicêmicos & $4,6 \pm 6,7$ & $4,4 \pm 5,0$ \\
Dose de insulina humana N (U/dia) & $51,8 \pm 16,5$ & $49 \pm 13$ \\
Dose de insulina humana R (U/dia) & $13,5 \pm 9,0$ & $14,5 \pm 8,7$ \\
HbA 1 IC & $6,05 \% \pm 1,3 \%$ & $6,7 \% \pm 1,4 \%$ \\
(variação) & $(4,1 \%-8,1 \%)$ & $(4,6 \%-9,9 \%)$ \\
\hline
\end{tabular}

O grupo 1 iniciou o estudo com insulina regular e o grupo 2 com insulina lispro

* A idade está representada como mediana.

* Não houve diferenças significantes em todos os parâmetros avaliados entre os grupos.

Tabela 2 - Doses médias de insulina regular e lispro utilizadas durante o decorrer do estudo nos 2 grupos de estudo.

\begin{tabular}{lcc}
\hline & Grupo 1 & Grupo 2 \\
\hline $\begin{array}{l}\text { Dose da insulina regular (U/dia) } \\
\text { Inicio }\end{array}$ & $14,5 \pm 8,7$ & $13,5 \pm 13$ \\
Final & $15,5 \pm 8,0$ & $18,0 \pm 8,0$ \\
& & \\
Dose da insulina lispro (U/dia) & $15,4 \pm 8,7$ & $13,5 \pm 9,0^{*}$ \\
Inicio & $15,0 \pm 8,0$ & $17,4 \pm 10$ \\
Final & & \\
\hline
\end{tabular}

${ }^{*} p=0,004$ em relação ao início do período.

Não houve diferença significante das doses de insulina regular ou lispro empregadas, quando os dois grupos foram comparados.

Grupo $1=$ uso inicial de insulina regular.

Grupo 2 = uso inicial de insulina lispro. 

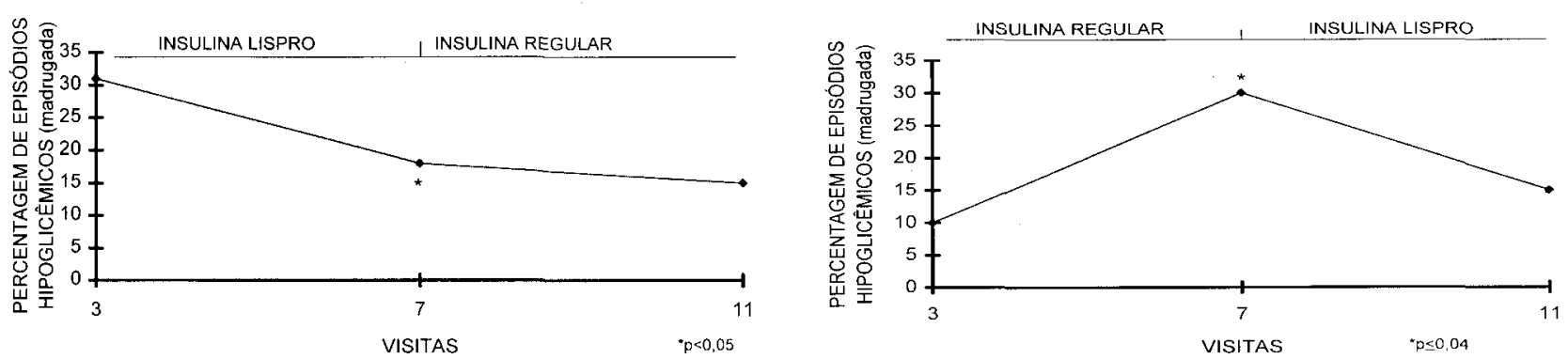

Figura 2 - Percentagem de episódios hipoglicêmicos no período da madrugada em pacientes em uso inicial de insulina lispro (A) ou insulina regular (B). A incidência de hipoglicemia na madrugada foi significantemente menor na fase do estudo em uso de insulina lispro.

neste grupo. A dose de insulina lispro ou regular não foi diferente comparando os 2 grupos. A freqüência de aplicação de insulina NPH durante todo o estudo foi de 1 a 2 vezes por dia; as insulinas regular e lispro foram aplicadas 2 a 3 vezes por dia.

Não houve variação na excursão de glicose no decorrer de todo estudo em qualquer dos grupos. O decréscimo percentual relativo da glicemia foi significantemente maior com insulina lispro no período do almoço na primeira fase do estudo $(p<0,02)$, não havendo diferença significante entre os grupos na segunda fase do estudo. Não houve variação da $\mathrm{HbA}_{1 \mathrm{c}}$ ao longo do estudo em qualquer dos grupos de tratamento $\left(\mathrm{HbA}_{\mathrm{lC}}\right.$ inicial $=6,7 \% \pm 1,4 \%$ vs. $\mathrm{HbA}_{1 \mathrm{C}}$ final $=6,6 \% \pm 1,5 \%$ no grupo $\mathrm{l}$ - uso inicial de insulina lispro e $\mathrm{HbA}_{1 \mathrm{C}}$ inicial $=6,05 \%$ $\pm 1,3 \%$ vs. $\mathrm{HbA}_{1 \mathrm{C}}$ final $=6,45 \% \pm 1,1 \%$ no grupo $2-$ uso inicial de insulina regular).

O número total de episódios hipoglicêmicos no decorrer de todo o estudo não foi diferente, comparando-se os grupos em uso de insulina lispro ou insulina regular. Entretanto, pacientes que iniciaram tratamento com insulina lispro apresentaram uma redução significante na freqüiência de episódios hipoglicêmicos nos períodos da noite e madrugada $(p<0,05)$, quando comparados com o período pré-randomização (Figura 2A). No grupo que iniciou com insulina regular houve incremento da freqüência de hipoglicemia na madrugada ( $\mathrm{p}=0,038)$ na primeira fase do estudo, com redução dos episódios hipoglicêmicos na segunda fase do estudo com insulina lispro ( $p=0,04$; Figura $2 B$ ).

\section{DADOS DE SEGURANÇA}

Houve um incremento significante no peso corporal dos pacientes na primeira fase do estudo, independente do grupo de tratamento alocado, não havendo diferença entre os 2 grupos (ganho médio de peso de $1,5 \mathrm{~kg}$ nos 2 grupos; $\mathrm{p}<0,02)$. Este parâmetro se manteve estável na segunda fase do estudo. Não houve diferença em relação aos sinais vitais (freqüência cardíaca e níveis de pressão arterial) durante o estudo.

Ao final do estudo, os pacientes foram questionados a respeito do esquema terapêutico de sua preferência, e $68 \%$ dos pacientes preferiram o uso de insulina lispro em comparação à insulina regular, referindo maior comodidade de uso.

\section{DISCUSSÃO}

O objetivo primário deste estudo foi comparar o efeito da insulina lispro e insulina humana regular no controle glicêmico, particularmente pós-prandial e na $\mathrm{HbA}_{\mathrm{lc}}$. Não conseguimos detectar neste estudo difcrença entre os grupos em relação aos resultados de excursão da glicose ou $\mathrm{HbA}_{\mathrm{IC}}$. Na primeira fase do estudo, o decréscimo percentual relativo da glicemia foi significantemente maior no grupo que estava em uso de lispro quando comparado com o grupo $\mathrm{cm}$ uso de insulina regular. $\mathrm{Na}$ segunda fase do estudo se perdeu esta diferença, provavelmente devido a não adequação da dose da insulina lispro e/ou insulina basal.

Vários estudos prévios demonstraram que o uso de insulina lispro leva a uma redução significante da glicemia pós-prandial quando comparado a insulina regular (17-25), e alguns estudos também mostraram uma redução significante da $\mathrm{HbA}_{1 \mathrm{C}} \mathrm{em}$ uso de insulina lispro (30-33). Estes estudos sugerem que, quando os pacientes são transferidos para insulina lispro, a estratégia de tratamento deve ser de ajustar tanto os esquemas de insulinização pré-prandiais quanto $o$ csquema de doses e freqüência da insulina basal (3134). Esta estratégia melhoraria o controle glicêmico global sem aumentar o risco de hipoglicemia (31). Em nosso estudo, é possível que não tenhamos evidenciado uma mudança significante no controle glicêmico, provavelmente por insuficiência de dose e freqüência do esquema de insulina basal, que foi mantida constante. Além disso, em nosso estudo as variações dos 
valores de glicemia foram muito amplas, tornando difícil se obter uma diferença estatística absoluta significante. O número de pacientes também pode ter sido insuficiente para se mostrar diferenças.

Alguns estudos sugerem que o conteúdo da refeição também possa ser importante no resultado final do controle glicêmico com insulina lispro (35-37). Os pacientes em nosso estudo fizeram uso de insulina lispro imediatamente antes das refeições e este horário de administração tem se mostrado mais eficaz do que qualquer esquema de insulina regular humana em controlar a glicemia pós-prandial, não estando associado a eventos hipoglicêmicos precoces ou tardios (28).

Avaliamos também a segurança do uso de insulina lispro, particularmente referente à taxa de hipoglicemia. A hipoglicemia é evento adverso comum da terapêutica insulínica, especialmente quando se adota o esquema de terapia intensiva, que pode resultar em um aumento na incidência de hipoglicemia severa (2-4). O medo de apresentar hipoglicemia noturna também pode interferir com a aderência do paciente ao tratamento (2). Quando se analisou o período inteiro do estudo, não encontramos diferença entre os 2 grupos de pacientes em relação ao número total de episódios hipoglicêmicos. Entretanto, quando os grupos foram analisados individualmente, a incidência de hipoglicemia no período da madrugada foi menor quando os pacientes estavam $\mathrm{cm}$ uso de insulina lispro, quando comparado a insulina regular. No grupo que iniciou com insulina regular, houve um incremento na freqüiência de episódios hipoglicêmicos noturnos, provavelmente devido a uma tentativa de otimizar o controle glicêmico, conforme o desenho do protocolo. No grupo que iniciou com insulina lispro, apesar de ter havido um aumento significante da dose desta insulina de ação rápida na primeira fase do estudo, houve uma redução significante na freqüência de episódios hipoglicêmicos noturnos.

Estudos prévios demonstraram uma incidência menor de episódios hipoglicêmicos em pacientes em uso de insulina lispro, particularmente episódios noturnos (12-16). A redução da taxa de hipoglicemia noturna tem sido observada tanto em pacientes diabéticos tipo l (1316) quanto em pacientes diabéticos tipo 2 (20). Em uma meta-análise de hipoglicemia severa em diabéticos tipo 1 , a freqüência de hipoglicemia severa foi significantemente menor em pacientes em uso de insulina lispro, do que naqueles em uso de insulina regular (17).

Não houve também diferença significante entre tratamentos em relação a descontinuações ou eventos adversos. A maioria dos pacientes relatou preferência pelo uso de insulina lispro, quando comparado à insulina regular. Estudos prévios com insulina lispro também demonstraram preferência dos pacientes por insulina lispro, quando comparada à insulina humana regular e referem melhora da qualidade de vida dos pacientes $(38,39)$.

A insulina lispro se mostrou uma opção segura, mais fisiológica c conveniente para o paciente. Um controle adequado e ótimo do controle glicêmico deve ser atingido com insulina lispro através de otimização da dose e freqüência de administração, não só da insulina lispro como também da insulina basal. Além disso, o teor da refeição também pode ser importante para se atingir um controle glicêmico idcal.

\section{AGRADECIMENTOS}

Este estudo foi patrocinado pelo Laboratório Eli Lilly do Brasil.

\section{REFERÊNCIAS}

1. Diabetes Control and Complications Trial Research Group: The effect of intensive treatment of diabetes on the development and progression of long-term complications in insulin-dependent diabetes mellitus. N Engl J Med 1993:329:977-86.

2. Reichard P. A risk-benefit assessment of conventional versus intensive therapy. Drug Saf 1994;10: 196-202.

3. Hansen KF, Dahl-Jorgensen K, Lauritzen T, Feldt-Rasmussen B, Brichmann-Hanssen $O$, Deckert T. Diabetic control and microvascular complications: the near normoglycemic experience. Diabetologia 1986;29:677-85.

4. Reichard $P$, Nilsson B-Y, Rosenqvist U. The effect of longterm intensified insulin treatment on the development of microvascular complications of diabetes mellitus. $\mathbf{N}$ Engl J Med 1993;329:304-9.

5. Davey P. Grainger D, Macmillan J, Rajan N. Aristides M, Gliksman M. Clinical Outcomes with insulin lispro compared with human regular insulin: a meta-analysis. Clin Therap 1997; 19:656-74.

6. Howey DC, Bowsher RR, Brunelle RL, Woodworth JR. (Lys(B28), Pro(B29)) -human insulin: a rapidly absorbed analog of human regular insulin. Diabetes 1994:43:396-402.

7. Wilde MI, McTavish D. Insulin lispro. A review of its pharmacological properties and therapeutic use in the management of diabetes mellitus. Drugs 1997;54:597-614.

8. Torlone E, Fanelli C, Rambotti AM, Kassi G, Modarelli F, Di Vincenzo et al. Pharmacokinetics, pharmacodynamics and glucose counterregulation following subcutaneous injection of the monomeric insulin analogue (Lys(B28), Pro(B29)) in IDDM. Diabetologia 1994;37:713-20.

9. ter Braak EW, Bianchi R, Erkelens DW. Faster, shorter and more profound action of (Lys(B28), Pro(B29)) human regular insulin analogue compared to regular insulin irrespective of the injection site (Abstract). Diabetes 1993;42:207A.

10. Heinemann L, Starke AAR, Heding $L$, Jensen I, Berger $M$. Action profiles of fast insulin analogues. Diabetologia 1990:33:384-6.

11. Holleman F, Hoekstra JBL. Insulin lispro. N Engl J Med 1997; $337: 176-83$. 
12. Anderson JH, Brunelle RL, Koivisto VA, Trautmann ME, Vignati $L$, DiMarchi $R$, et al. Improved mealtime treatment of diabetes mellitus using an insulin analogue. Clin Therap 1997:19:62-72.

13. Anderson JH, Brunelle RL, Kolvisto VA, Ptutzner A, Trautman ME, Vignati $L \mathrm{Jr}$, et al. Reduction of postprandial hyperglycemia and frequency of hypoglycemia in IDDM patients on insulin analogue treatment. Multicenter Insulin Lispro Study Group. Diabetes 1997;46:265-70.

14. Holleman F, Schmitt H, Rottiers R, Rees A, Symanowski S, Anderon $\mathrm{JH}$ for the Benelux-UK insulin lispro study group. Reduced frequency of severe hypoglycemia and coma in well-controlled IDDM patients treated with insulin lispro. Diabetes Care 1997;20:1827-32.

15. Pfutzner A, Kustner E, Forst T, Schulze-Schleppinghoff $B$, Trautmann M. Haslbeck $M$, et al. On behalf of the German Insulin lispro/IDDM study group: Intensive insulin therapy with insulin lispro in patients with type 1 diabetes reduces the frequency of hypoglycemic episodes. Exp Clin Endocrinol 1996; 104:25-30.

16. Brunelle RL, Llewelyn J, Anderson JH, Gale EAM, Koivisto VA. Meta-analysis of the effect of insulin lispro on severe hypoglycemia in patients with type 1 diabetes. Diabetes Care 1998;21:1726-31.

17. Vignati L, Anderson JH, Iversen PW - Multicenter Insulin Lispro Study Group. Efficacy of insulin lispro in combination with NPH human insulin twice per day in patients with insulin-dependent or non-insulin-dependent diabetes mellitus. Clin Therap 1997: 19:1408-21.

18. Jacobs MAJM, Keulen ETP, Kanc K, Casteleijn S, Scheffer P, Devillé W, et al. Metabolic efficacy of preprandial administration of Lys(B28). Pro(B29) human insulin analog in IDDM patlents. A comparison with human regular insulin during a three-meal test period. Diabetes Care 1997:20:1279-86.

19. Campbell RK, Campbell LK, White JR. Insulin lispro: its role in the treatment of diabetes mellitus. Ann Pharmacother 1996;30: 1263-71.

20. Anderson JH, Brunelle RL, Keohane P, Koivisto VA, Trautmann ME, Vignati $L$, ef al. Mealtime treatment with insulin analog improves postprandial hyperglycemia and hypoglycemia in patients with non-insulin-dependent diabetes mellitus. Arch Inter Med 1997:157:1249-55.

21. Garg SK, Carmain JA, Braddy KC, Anderson JH, Vignati $L$, Jennings $M K$, et al. Pre-meal insulin analogue insulin lispro vs. Humulin $(B)$ insulin treatment in young subjects with type 1 diabetes. Diabetic Med 1996; 13:47-52.

22. Pampanelli S, Torlone E, Lalli C, Sindaco PD, Ciofetta M Lepore M. Improved postprandial metabolic control after subcutaneous injection of a short-acting insulin analog in IDDM of short duration with residual pancreatic $\beta$-cell function. Diabetes Care 1995;18:1452-9.

23. Feiglos MN. Thacker $\mathrm{CH}$, English J, Bethel MA, Lane JD. Modification of postprandial hyperglycemia with insulin lispro improves glucose control in patients with type 2 diabetes. Diabetes Care 1997:20:1539-42.

24. Torlone E, Pampanelli S, Lalli C. Del Sindaco P, Di Vicenzo A, Rambotti AM, et al. Effects of the short-acting insulin analog (Lys (B28), Pro(B29)) on postprandial blood glucose control in IDDM. Diabetes Care 1996; 19:945-52.

25. Jansson P-A, Ebeling P, Smith U, Conget I, Coves MJ, Gomis R. Improved glycemic control can be better maintained with insulin lispro than with regular insulin. Diab Nutr Metab 1998:11:194-9.

26. Howey DC, Bowsher RR, Brunelle RL, Rowe HM, Santa PF Downing-Shelton J. (Lys(B28), Pro(B29))-human insulin: effect of injection time on postprandial glycemia. Clin Pharmacol Ther 1995:58:459-69.

27. Rutledge KS, Chase $P$, Klingensmith GJ, Walravens $P A$ Slover RH, Garg SK. Effectiveness of postprandial humalog in toddlers with diabetes. Pediatrics 1997:100:968-72.

28. Schernthaner $G$, Wein W, Sandholzer K, Equiluz-Bruck $S$, Bates $P$. Birkett MA. Postprandial insulin lispro. A new therapeutic option for type 1 diabetic patients. Diabetes Care 1998:21:570-3

29. Rami B, Schober E. Postprandial glycaemia after regular and lispro insulin in children and adolescents with diabetes. Eur J Pediatr 1997:156:838-40.

30. Melki V, Renard E, Lassmann-Vague V, Boivin S, Guerci B, Hanaire-Broutin $\mathrm{H}$. Improvement of $\mathrm{HbA} 1 \mathrm{C}$ and blood glucose stability in IDDM patients treated with lispro insulin analog in external pumps. Diabetes Care 1998;21:977-82.

31. Ebeling P, Jansson PA, Smith U, Lalli C, Bolli GB, Koivisto $V A$. Strategies toward improved control during insulin lispro therapy in IDDM. Importance of basal insulin. Diabetes Care 1997;20: 1287-9

32. Ahmed AB, Mallias J, Home PD. Optimization of evening insulin dose in patients using the short-acting insulin analog lispro. Diabetes Care 1998;21:1 162-6.

33. Ahmed ABE, Home PD. Optimal provision of daytime $\mathrm{NPH}$ insulin in patients using the insulin analog lispro. Diabetes Care 1998:21:1707-13.

34. Sindako PD, Ciofetta M, Lalli C, Perriello, Pampanelli, Torione $E$, et al. Use of the short-acting insulin analogue lispro in intensive treatment of type 1 diabetes mellitus: importance of appropriate replacement of basal insulin and time-interval injection-meal. Diabet Medicine 1998;15:592-600.

35. Burge MR, Castillo KR, Schade DS. Meal composition is a determinant of lispro-induced hypoglycemia in IDDM. Diabetes Care 1997:20:152-5.

36. Ronnemaa T, Vilkari J. Reducing snacks when switching from conventional soluble to lispro insulin treatment: effects on glycaemic control and hypoglycemia. Diabet Med 1998; 15:601-7.

37. Strachan MWJ, Frier BM. Optimal time of administration of insulin lispro. Importance of meal composition. Diabetes Care 1998:21:26-31.

38. Kotsanos JG, Vignati L, Huster W, Andreajasich C, Boggs MB, Jacobson AM, et al. Health-related quality-of-life resuits from multinational clinical trials of insulin lispro. Diabetes Care 1997:20:948-58.

39. Reviriego J, Millan M. Health-related quality of life and insulin lispro. A naturalistic approach. Diabetes Care $1998 ; 21: 1203-4$

\section{Endereço para correspondência:}

Marcia Jeha Kayath

Eli Lilly do Brasil

Av. Morumbi 8264

04703-002 São Paulo, SP

e-mail: kayath_marcia_jeha@|illy.com 